\title{
Requirements Elicitation and Prototyping of a Fully Immersive Virtual Reality Gaming System for Upper Limb Stroke Rehabilitation in Saudi Arabia
}

\author{
Maram AlMousa, ${ }^{1}$ Hend S. Al-Khalifa, ${ }^{1}$ and Hana AlSobayel ${ }^{2}$ \\ ${ }^{1}$ Information Technology Department, College of Computer and Information Sciences, King Saud University, Riyadh, Saudi Arabia \\ ${ }^{2}$ Department of Rehabilitation Sciences, College of Applied Medical Sciences, King Saud University, Riyadh, Saudi Arabia
}

Correspondence should be addressed to Hend S. Al-Khalifa; hendk@ksu.edu.sa

Received 3 March 2017; Revised 24 April 2017; Accepted 9 May 2017; Published 7 June 2017

Academic Editor: Miltiadis D. Lytras

Copyright (C) 2017 Maram AlMousa et al. This is an open access article distributed under the Creative Commons Attribution License, which permits unrestricted use, distribution, and reproduction in any medium, provided the original work is properly cited.

Stroke rehabilitation plays an important role in recovering the lifestyle of stroke survivors. Although existing research proved the effectiveness and engagement of nonimmersive virtual reality- (VR-) based rehabilitation systems, limited research is available on the applicability of fully immersive VR-based rehabilitation systems. In this paper, we present the elicited requirements of a fully immersive VR-based rehabilitation system that will be designed for domestic upper limb stroke patients; we will also provide an initial conceptual prototype of the proposed system.

\section{Introduction and Background}

Stroke disease is classified as the third cause of death in the world after heart and cancer diseases [1]. According to the World Health Organization, there are 15 million people who suffer from stroke worldwide each year [2]. About five million of these people die, while 5 million become permanently disabled. Nation wise, statistics indicate that more than 100 strokes occur daily in the Kingdom of Saudi Arabia [3]. Many experts expect that the rate of stroke will rapidly grow as a result of the increasing population ages, occurrence of high blood pressure, number of smokers, incidence of diabetes, and obesity [4]. Many survivors of stroke suffer from residual disabilities and reduced quality of life [5], particularly upper limb residual hemiparesis [6].

These facts reveal the urgent need for rehabilitation programs that facilitate recovering daily life activities for stroke survivors for better quality of life [1]. This helps them to relearn basic skills such as eating, dressing, and walking, which were lost due to the damage of stroke [6].

Rehabilitation is generally performed in hospitals in the early stages after stroke [7]. As the treatment advances, patients often need to go to specialized units for a supervised outpatient therapy. Finally, the last stage consists of homebased programs that may involve the professionals' visits in order to permit the patient to develop his/her skills in home environment [8].

The tests of rehabilitation programs suggest that the patient's function recovers by performing a series of repeated exercises identified with a certain goal [9]. However, these programs usually lack patient motivation for performing several repetitive tasks at regular intervals. Moreover, healthcare providers need to ensure that this treatment program is completed as outpatients [8].

Therefore, the focus of recent research has been on virtual reality- (VR-) based rehabilitation systems, as they have been shown to be more effective and engaging than conventional rehabilitation therapy. However, most of these systems are nonimmersive. A recent Cochrane review, looking at using VR in stroke rehabilitation, showed growing evidence on the benefits of VR and interactive video gaming on improving upper limb function and activities of daily living function when used as an adjunct to the usual rehabilitation program. However, the clinical trials that are so far available did not have sufficient power to reach a conclusion on the effectiveness of VR on upper limb performance among people 
with stroke [5]. The lack of sufficient evidence warrants more research on this area. Therefore, our contribution in this paper contains three parts:

(1) An extensive state-of-the-art review of the field of virtual reality and serious games for upper limb stroke rehabilitation: the review shows the gaps in the literature in terms of technologies used and employed approaches. To overcome these issues, recent research has been focusing on virtual reality- (VR-) based rehabilitation systems, as they were proven to be much effective and engaging compared to conventional rehabilitation therapy. However, most of these systems are nonimmersive, despite the fact that fullimmersive systems may have the potential to provide a high level of user engagement that would solve the lack of patient interest issue, which our current proposed system will overcome.

(2) Design guidelines: we present a list of elicited requirements to help developers in designing fully immersive virtual reality systems for upper limb stroke rehabilitation.

(3) Mobility: the proposed rehabilitation system is of low cost, portable, and easy to set up and can be accessed remotely by clinicians to monitor patients' progress. This will, in fact, provide a mobile rehabilitation system that can be used both at the clinic and at the patient home.

The rest of the paper is organized as follows: Section 2 highlights some previous work in the field of VR and games for stroke rehabilitation. Section 3 presents the method followed in this project. Section 4 presents a detailed design of the proposed VR system prototype and its requirements elicitation. Finally, Section 5 concludes the paper with future work.

\section{Stroke Rehabilitation Games}

The existing stroke rehabilitation games can be classified into two classes: commercial off-the-shelf games and customdesigned rehabilitation games. The custom-designed rehabilitation games can be further classified into nonimmersive and fully immersive VR systems. Next a thorough discussion about previous research that used the above-mentioned types is presented.

2.1. Off-the-Shelf VR Games. In the early days of motor rehabilitation, researchers explored the use of the available off-the-shelf rehabilitation video games. In 2010, a study was conducted to evaluate the effectiveness of using Wii technology for the rehabilitation of stroke [10]. They compared the feasibility, safety, and efficacy of acquiring Wii commercial games such as Wii Sports and Cooking Mama for stroke rehabilitation versus the recreational therapy such as bingo and playing cards, on two groups of eleven stroke patients for two months. The results of their evaluation revealed that the Wii gaming system group spent considerably less session time than the recreational therapy group. Moreover, the Wii gaming system group showed better improvements in motor function than the recreational therapy group.

Another research by Rand et al. [11] examined the feasibility of using Sony PlayStation 2 EyeToy commercial games. They evaluated the Wishy-Washy and Kung-Foo video games with both stroke patients and healthy individuals. The results showed that, despite the fact that both groups enjoyed the games, the stroke patients faced difficulties and required assistance from the therapist to complete these games. In fact, two patients became frustrated as they could not manage to interact with the games. This problem revealed the need for developing a specialized rehabilitation game that fits stroke rehabilitation requirements and stroke patients' capabilities.

Before moving to the next class of VR-based rehabilitation games, we will present an interesting, useful, and very recent study on the requirements that stroke patients look for in game-based rehabilitation [12]. This study was conducted to encourage patients to complete their rehabilitation practices at home, as well as to identify and eliminate the barriers in performing game-based rehabilitation at home. The researchers interviewed 30 patients and 19 therapists to get a better understanding of the current exercises, challenges, and expectations on game-based rehabilitation systems. Furthermore, they performed a gaming experiment with 14 therapists to observe how they select commercial games that are compatible for rehabilitation and what parameters they look for in these games, to make it a rule when designing such a system. Twenty-four commercial games such as Block Puzzle, Angry Birds, and Fruits Slice were selected based on the therapists' recommendations. Afterwards, these games were evaluated randomly by at least 5 other therapists. At the end of their study, they came up with interesting and useful findings. The findings are summarized in that the traditional rehabilitation practices are less effective, the current game-based rehabilitation systems lack excitement qualities, patients prefer to use game-based rehabilitation systems built upon existing video games, and patients prefer to interact with game-based rehabilitation systems with large displays using physical sensors. Finally, they suggested three recommendations to be considered when designing or selecting a game-based rehabilitation system. Firstly, the game-based rehabilitation system should have sufficient logic challenge to attract the patients' interests. Secondly, the system should be user-driven which means that the user takes the initiative and the gameplay is not restricted in time to prevent users from feeling nervous. Thirdly, the system should be designed with intuitive and simple interface. However, the main drawback in their study is that they did not evaluate the selected game on actual stroke patients other than their therapists.

2.2. Custom-Designed Rehabilitation Games. To overcome the limitations of off-the-shelf video games, many researchers proposed implementing a specialized game that suits the needs of stroke patients and the stroke rehabilitation process, which has become the focus of recent research studies.

2.2.1. Nonimmersive VR-Based Rehabilitation Systems. Much research has been done on this subject. For instance, the University of Ulster conducted numerous researches and 




(a)



(b)

FIgURE 1: Rabbit Chase game (a) and Arrow Attack game (b) [13].

applications for stroke rehabilitation games. They explored developing and evaluating both nonimmersive and immersive VR-based rehabilitation systems. They first developed several immersive systems in 2007; then, in 2009, the same research group has published another research containing a description of several nonimmersive stroke rehabilitation games which tend to be of low cost and more suitable for home-based therapy [13]. The proposed games used the standard webcam technology in order to capture video data of users' movement. The hand movements were then tracked by wearing gloves or holding objects of single consistent color (the marker) that is easily distinguished by the webcam. Interestingly, the patients are allowed to sit or stand when playing the game. The first webcam game is Rabbit Chase which must be played using only one marker on either hand. The goal of this game is to chase and catch rabbits as they appear from one of the four holes displayed on the screen as shown in Figure 1(a).

The second game is called Arrow Attack which displays two arrows on the screen, one pointing to the left and the other pointing to the right which move among 4 boxes shown on screen. The patient should try to touch the two arrows at the same time when they reach each box, using the correct hand, as shown in Figure 1(b). Moreover, they have developed a Nintendo Wii remote game called vibraphone [7]. This game permits the patient to play a virtual vibraphone instrument using Nintendo Wii remote controllers.

A recent study in 2015 introduced three games that provide a rehabilitation of the upper limb following stroke [14]. Their aim is to investigate the adherence to home-based rehabilitation when using VR games. Their first game is called Spacerace which lets users navigate a spacecraft through some barriers. The second game is called Spongeball which lets users hit a target with a virtual ball as shown in Figure 2.

Finally, the third game is called Ballonpop which lets users pop virtual balloons. These games have the ability of changing the level of difficulty according to the users' performance. Moreover, all these games require the user to use a glove that is designed and created by the research. This glove is tracked by one or two Nintendo Wii remotes that are connected to the computer which the game is running on. They have evaluated the use of the developed rehabilitation



FIGURE 2: Spongeball game [14].

games at home on 17 participants for 8 weeks. Results proved that the participants valued the flexibility and motivation of this type of therapy. However, they faced some difficulties of getting used to the equipment.

Furthermore, the researchers in [15] highlighted a number of limitations of using commercial games for rehabilitation prior to presenting their proposed system. These limitations are as follows: the difficulty level of the games is extremely high and challenging to the patients' abilities, especially as this difficulty level cannot be adjusted in most games. Moreover, as the games are very challenging to the patients, they might get negative scores and feedback, which would be frustrating and depressing for them. For this purpose, they decided to develop and evaluate four customdesigned rehabilitation games. They employed a low-cost depth-sensing camera to enable users to interact with the game's virtual objects without the need for controllers or body-worn sensors. The first game is Ball Maze that requires users to direct the ball around the maze into the hole using leaning movements (forward, backward, left, and right) of the torso. The second game is Fridge Frenzy that requires users to hit as much as possible of milk cartons by moving the torso side to side. The third game is Tentacle Dash that requires users to avoid getting hit by a bunch of tentacles through leaning and side stepping the torso. Finally, the fourth game is Bubble Fish that requires users to shoot bubble in order 




(a)



(c)



(b)



(d)

FIGURE 3: Games screenshots [15].

to hit the fish using their arm. Screenshots of the games are presented in Figure 3.

The results of their study revealed that most of the participants enjoyed the developed games and considered them helpful, and they were willing to continue using them in their therapy. Furthermore, the participants have shown great improvements in several functional outcome measures. Gladly, there were no serious adverse safety events reported, except a minor increase in pain for most participants.

Another study was conducted in Hanyang University to develop and assess the usability and clinical efficacy of a task-specific interactive VR-based rehabilitation system for the upper extremity of stroke patients, named RehabMaster [16]. They used a Kinect-like depth camera to track the user's movement. Their system consists of four components: a user management component that holds the patients' information; an assessment component that tracks the rehabilitation progress of the patient through using the following outcome measures: Fugl-Meyer Assessment, Action Reach Arm Test, and Motricity Index; a rehabilitation training component that enables patients to practice some arm exercises through copying the avatar movements; and rehabilitation games component that enables patients to perform exercises through engaging games. There are four different types of games in the last component. The first game is called Underwater Fire where the user is required to shoot the fish using two weapons. The second game is called Goalkeeper where the user is required to catch the football. The third game is called Bug Hunter where the user is required to catch the bug on display. Finally, the fourth game is called Rollercoaster where the user is required to copy the postures of the displayed avatar. Screenshots of these games are presented in Figure 4.
The difficulty level of these games is controlled by the occupational therapist. The researchers performed a usability test before conducting the clinical trial to better improve their system. Afterwards, they performed two clinical trials. In the first trial, seven participants were recruited to use the RehabMaster system daily for 30 mins in two weeks. The second trail was randomized and controlled in which two groups of 16 participants received either a conventional therapy or a RehabMaster with conventional therapy in 10 sessions. The result of their study showed that the patients in the RehabMaster group have greater improvements in FuglMeyer Assessment (FMA) score than the other group, while the improvements in Modified Barthel Index (MBI) were almost the same.

Apparently, all the previously cited stroke rehabilitation systems did not incorporate the latest advanced technologies such as the head mounted displays that could provide a better high-end virtual reality experience and hence higher patients' engagement. However, there has been a relatively little research on developing such games, which we will discuss in the next section.

\subsubsection{Immersive VR-Based Rehabilitation Systems. As has} been mentioned earlier on the immersive rehabilitation game that was proposed by the University of Ulster in 2007, a research group developed an immersive VR game that incorporates the use of HMD, named Catch-the-Orange [17]. They have used regular input devices such as keyboard and mouse for the operator and several VR input hardware Ascension MotionStar wireless magnetic sensors which capture the patient's hand, arm, and upper body movements. The output devices include a desktop computer for the operator and 




Underwater Fire game

Goalkeeper game



Bug Hunter game

Rollercoaster game

FIGURE 4: Games screenshots [16].



(a)



(b)

FIGURE 5: Catch-the-Orange game (a) and user playing the game (b) [17].

the HMD Virtual Research 1280 stereo for patients. In this game, the patient is required to hold a virtual basket and is challenged to catch the falling oranges before reaching the ground as presented in Figure 5. A sensor is embedded in the real basket to control the position and orientation of virtual basket. The problem with this research is that the used hardware devices were not suitable for home-based therapy.

Another study that provides a fully immersive rehabilitation game for stroke survivors was conducted in the University of California using the Oculus Rift HMD [18]. The researchers incorporated Oculus Rift with Kinect and a pair of haptic gloves. The Kinect was used to track the user body's movement, while the gloves track his/her fingers' movements. Their game consists of two scenes. The first scene requires the user to grab four cylinders located around him and move them into a box. In this scene, the user's position data and the time (s)he spends to move the object into the box are recorded. The second scene is more like an exercise than a game. It requires the user to follow instructions such as raising his/her hand as high as possible and then record these data. Their game was evaluated by one stroke patient who had stroke 4 years ago. Overall, the patient enjoyed their game and would recommend it for others. However, these abovementioned systems are hard to set up and afford.

2.3. Discussion. In conclusion the previous studies on stroke rehabilitation systems indicated that virtual reality technology can improve patients' motor function, increase their motivation, and provide a great solution for home-based therapy.

We have noticed from the previous work that different VR technologies from different brands have been incorporated in such systems. The hardware that was mostly used in these systems was the commercial devices: Wii technology and Kinect, perhaps, because Wii SDKs are publicly opened for own application development and the Kinect offers full body tracking without a controller with reasonable cost [19].

Many of these rehabilitation systems provide a set of games to combine a variety of rehabilitation exercises and make the system more diverse and interesting. Moreover, 
most of these systems offer multiple levels of difficulty to suit many patients who are high and low functioning.

Also, we have concluded from the previous studies that several VR-based rehabilitation systems provide recoding patient's movement data to enable the therapist to track his/her patient recovery progress. However, few of them allow monitoring these data remotely, which make these systems less suitable for home-based rehabilitation.

Generally speaking, the first class of VR-based rehabilitation systems $[7,8]$ has several drawbacks such as the inappropriate difficulty level for stroke patients' capabilities in which they cannot use it without assistance and the lack of recording and reporting features, although it provides high engagement and excitement qualities as stated in [9], as it was designed mainly for that purpose.

The second class $[5,7,14,15]$ has been shown to be a more suitable solution as it offers the following features:

(i) Providing several difficulty levels that are adjusted automatically.

(ii) Recording the patient's performance information to be monitored by the therapists.

(iii) Presenting positive feedback.

Yet, only a few number of studies have been done on immersive VR-based rehabilitation systems [17, 18], which may have the potential to provide a high level of user engagement that would solve the repetitive nature issue of rehabilitation tasks. Furthermore, to the best of our knowledge, none of these systems was provided in Arabic language or has been examined in Saudi Arabia environment. Therefore, the purpose of our study is to develop a portable and fully immersive VR-based rehabilitation system designed for domestic upper limb stroke patients. The system will allow the patients to perform the rehabilitation tasks more excitingly and will enable the healthcare providers to track the patients' recovery progress remotely to suit the requirements of home-based rehabilitation. The proposed system will incorporate the use of Oculus Rift head mounted display (HMD) and the Leap Motion hand tracker to achieve the highest level of patient excitement. We present in this paper the developmental phase of the system.

\section{Materials and Methods}

This project is divided into two phases: The first phase includes system design. The second phase, which will be reported later, will include the development and evaluation of the system feasibility and usability. In this paper we will report on the first phase of the project, which includes needs assessment and system design.

3.1. Needs Assessment. We aimed at gathering information and building a better understanding of patients with stroke, their needs, and the stroke rehabilitation process in order to determine the proposed rehabilitation system contents and functions. This was attained through two main steps: (1) distilling the recommendation and best practices from previous work and (2) interviewing the specialists (occupational therapists (OT) and physical therapists (PT)), which included (i) observing and interviewing the therapists and patients during the rehabilitation session,

(ii) conducting a focus group with therapists in two major rehabilitation hospitals in Riyadh, to identify their perspectives on the requirements for the proposed game.

We visited two medical centers: King Fahad Medical City (KFMC) and Sultan Bin Abdulaziz Humanitarian City (SBAHC). These centers were chosen since they are the leading hospitals in the rehabilitation field in Saudi Arabia. Moreover, they are considered as the largest and most advanced medical centers in the middle east region [20,21].

KFMC contains a hospital which was the first hospital that offers comprehensive services for rehabilitation of recently injured patients and patients from all ages who need rehabilitation therapy. Moreover, it is the first medical center that was accredited by the Commission on Accreditation of Rehabilitation Facilities (CARF) outside North America. Nowadays, KFMC is a member in CARF that can assess other hospitals in Europe and United State.

SBAHC is a nonprofit institution specialized in rehabilitation services. It employs the latest medical technologies to serve its patients from all ages, with a very knowledgeable medical crew and a total capacity of 462 beds. SBAHC has obtained both the Joint Commission International (JCI) accreditation and CARF accreditation.

There were multiple visits to both centers. The first visit included conducting a focus group with a number of specialists from both centers where several questions were raised and answered. These questions were about the stroke patient's characteristics, the processes and approaches of the rehabilitation therapy, the rehabilitation sessions for inpatients and outpatients, the upper limb rehabilitation exercises, the assessment and evaluation outcome measures, and the VR-based rehabilitation system requirements.

3.2. Needs Assessment Results. The focus group lasted for about one hour in each medical city. The result of these focus groups is summarized in the following points:

(i) The common patterns of recovery for stroke patients are lower limb, then body balance, and then upper limb. The upper limb recovery is considered more challenging than the lower limb because it takes more time and effort to recover. This is due to the fact that the hand movements are done in space unlike the leg movements which move on a solid ground. Besides, the hand movement varies more than the leg movement.

(ii) The processes of treating a stroke patient in KFMC and SBAHC are as follows: checking if the patient meets certain eligibility criteria; if yes (s)he will stay in the hospital; then all PTs and OTs will gather to evaluate his/her case to decide the period of stay and goals towards function (e.g., walking by his/her own, eating by himself/herself); then the patient will receive therapy for one hour daily except in weekends; later his/her recovery progress will be evaluated to 
check if goals were reached or not and then determine whether the patient needs to continue staying at the hospital or can continue rehab at home; finally the follow-up visit is done for outpatients.

(iii) There are different types of approaches used for stroke rehabilitation, which are chosen depending on the patient capabilities. These types are strength training, function training, graded motor imagery using mirror, Constant Induced Movement Therapy (CIMT) by forcing the affected side, video gaming, sensory retraining, and mental practice.

(iv) The exercise tasks of upper limb stroke rehabilitation are similar to the hands movements of daily life activities. However, they can be broken down into the following movements:

(a) Moving hand towards face or mouth.

(b) Moving the shoulder (raising hand) to 120 degrees or less where the normal degree is 170 .

(c) Grasping object.

(d) Releasing object.

(e) Elbow extension and flexion.

(v) The specialists proposed the following suggestions for the system design: the system should be related to culture, engaging, affordable, easy to set up, and easy to use, include patient authentication feature to prevent cheating by allowing others to do the patient's work, display warning when the quality of movement is wrong, be in Arabic language, have fonts and images being big and clear, and be preferred to be used without Internet connection.

(vi) The therapists want the records to contain the following information: the performed exercises, the number of repetitions of each exercise, distance or degree of movement, quality of movement if possible, and spent time.

The second visit involved on-site observation of patients, by OTs and PTs during the rehabilitation session, which included interviewing the OTs and PTs, as well. This observation lasted for four hours in SBAHC. It was important to gather reliable information, take notes, and get a clearer view of what are the actual rehabilitation therapy activities and environment. The observation visit's findings are summarized in the following:

(i) Most of the patients need to do some exercises to flex their muscles and warm up in the beginning of the rehabilitations session.

(ii) Patients usually take a break after each exercise.

(iii) OTs struggle to keep some patients motivated and focused on the rehabilitation exercises.

(iv) The patients who use the conventional game seem more interested and tend to finish their therapy faster than the patients who use exercise devices such as bicycle. (v) Typically, the session takes from one hour to a maximum of two hours and a half. However, some patients cannot do more than forty-five or thirty minutes.

(vi) VR-based rehabilitation systems have been used recently in SBAHC. However, these systems are nonimmersive, expensive, and large and difficult to move; require a therapist to set up and operate; and lack the remote monitoring feature, which makes them unsuitable for home-based rehabilitation.

\section{System Design}

Based on extensive literature review and considering the results of the needs assessment, the system's high-level architecture and the game main elements were designed.

At a high level, our system consists of three main components: the input and output devices (Oculus Rift [22], Leap Motion [23]), the game module, and the monitoring website.

The Oculus Rift is a head mounted display that tracks the movement of the user's head and immerses the user through displayed 3D images with a wide field of view. It was chosen for this system for the reason that it is wearable, affordable, and portable and can provide a high level of immersion that will keep the stroke patient engaged to continue the rehabilitation exercises. The Leap Motion is a hand-tracking device that can be used without a controller or gloves but only with bare hands. It was chosen for this system for the reason that it can be attached to the Oculus Rift HMD and more importantly it can allow patients who have not regained sufficient hand power to use a game controller to interact with the system. Moreover, the two devices would provide patients with high-end virtual reality experience that is cost-effective. The game module will be developed using the game engine Unity [24]. The game will require users to perform exercises that are inspired by the rehabilitation therapy techniques. The movements to be included in the game were provided by OTs and PTs of Sultan Bin Abdulaziz Humanitarian City (SBAHC) and King Fahad Medical Center (KFMC). The game module will record data regarding the users' performance into the system's database that can be viewed in a web browser to be accessed remotely by the therapist. Figure 6 illustrates our system architecture.

4.1. User Characteristics. Based on our previous observations, our proposed system will be designed to serve the following two user groups:

(1) Stroke patients who need to complete their upper limb rehabilitation therapy: the system can serve both the inpatients at the hospital and outpatients at their home; these patients are expected to have minimum arm functional abilities to be able to interact with the rehabilitation game. According to the specialists from KFMC and SBAHC, the majority of stroke patients in Saudi Arabia are males; it is more common in people aged 45 and over, where two-thirds of them are above 65 as reported in [1] (however there exist some few cases with younger ages such as 18 or 30 years), and 




FIGURE 6: The system architecture.

native Arabic speakers; their educational background varies from undergraduates to professors.

(2) Therapists who want to remotely monitor their outpatients' recovery progress.

4.1.1. Personas. After we determined the characteristics of our system's users, we started designing the personas. Personas are imaginary characters created for different user types to help the system designers further understand the users' needs and preferences and to keep realistic ideas about the users throughout the project lifecycle. It is considered as a part of the User-Centered Design (UCD) framework that gives a great attention to the needs, desires, and limitations of end users at each stage of the system design. We developed personas representing the two user groups: patients with stroke and therapists.

First Persona. Hamad is a 62-year-old man. He is married and a father of five adult children. He is living in Riyadh with his wife and his youngest daughter. He likes to spend his time at home with his family. More importantly, Hamad truly cares about his health status and continuing his rehabilitation therapy at home, to improve his functional ability and become self-independent, in addition to his outpatient program. However, he constantly struggles to visit his rehabilitation hospital periodically, as it is far from his house, in addition to the fact that he cannot drive in his health situation. He needs a way to perform the rehabilitation tasks at home while being monitored by the therapists.

\section{Design Implications}

(i) The system should be affordable and easy to set up.

(ii) The system should be easy to use and learn.

(iii) The system should be accessible for elderly persons.

(iv) The system interfaces should be in the Arabic language.

(v) The system should provide clear and useful tutorials.

Second Persona. Saad is a 56-year-old man. He is married and a father of four children. He is a social person who likes to be around others and enjoys having conversations with others. More importantly, Saad is an inpatient who stays in the hospital with his son. He constantly struggles to continue his rehabilitation exercises during the rehabilitation session, since they are very boring and repetitive. Therefore, he usually interrupts them and chats with others instead.

Design Implications. Besides the design implications from the first persona, we would suggest the following:

(i) The system should grab the user's attention and engage him to encourage him to complete his rehabilitation tasks.

(ii) The system should provide adjustable levels of difficulty to provide an appropriate level of challenge.

Third Persona. Khalid is 31 years old. He works as an occupational therapist in one of the rehabilitation hospitals in Riyadh. He spends most of his time at work. He seeks to help and serve his patients to the maximum of his extent. Nevertheless, Khalid struggles with following up with his outpatients' progress reports, as their reports are not accurate or trusted. He needs a more reliable and professional way of tracking his outpatients' rehabilitation progress.

\section{Design Implications}

(i) The system should save records of the patient's movement data.

(ii) The system should allow the therapist to access these records remotely.

4.2. System Requirements. This section presents specification of the functional requirements for both the game module and the monitoring website.

\subsubsection{Game Module Functional Requirements}

(1) Arabic Language. The system interfaces should be in Arabic to avoid inconveniences, as it is the native language of most of the patients in Saudi Arabia.

(2) Comfortable Atmosphere to Enhance the User Experience. The system should provide a warm, bright, and natural environment with comfy sounds that are not loud or annoying to eliminate user frustration.

(3) Elderly Accessible. The system should use big images and font size that are readable by elderly people, in addition to providing consistent navigation and labeling. 
(4) The system should provide warm-up exercises option that enables the patient to warm up before starting the game.

(5) The game should be engaging and culture related to motivate the patient to complete the required exercises.

(6) The system should require the patient to do movements that are inspired by the rehabilitation therapy exercises.

(7) Tutorial. The system should display an animation that explains to the patient how they accomplish the game's tasks before starting the game.

(8) The system should provide various difficulty levels to suit the patients who have either low or high functioning capabilities and to provide an appropriate level of challenge that will keep them motivated.

(9) The system should adjust the difficulty level automatically according to the patient's performance to reduce the OT involvement.

(10) The system should enable the patient to use both hands to achieve the game's goal or restrict him/her to use one hand (the affected hand).

(11) The system should provide positive feedback with a score to encourage the patient to complete the rehabilitation game.

(12) The system should display a warning message before starting the game to inform the patient and his/her family members about the importance of completing the game's tasks by himself/herself to prevent cheating.

(13) The system should save records of the patient's movement data. The system should display these data to the patient with a simple language to permit the patient to understand his/her performance.

\subsubsection{Monitoring Website Functional Requirements}

(1) The system interfaces should be in English for the therapists.

(2) The system should allow the therapist to access and view his/her patient's movement data remotely in order to keep track of his/her patient's recovery progress.

4.3. Game Module. The game module is the heart of our system that contains the core functions of the system. The main user interface of our game module is comprised of five main options:

(1) Warm-up exercise option that enables the patient to warm up before starting the game.

(2) Rehabilitation game option that allows the patient to perform the rehabilitation exercise in an engaging form.

(3) User statistics option that contains a history of users playing records.
(4) Synchronization option that transfers the user statistics data to be seen by his/her therapist.

(5) Settings option that contains several game settings.

Once the user starts the game module for the first time, (s)he will be asked to enter his/her data such as ID (e.g., the patient medical record number), name, and birth date. Besides, a warning and welcoming message will be presented that gently alerts the patient and his/her family members of the importance of performing the rehabilitation game's exercise by him/her own.

In detail, the warm-up exercise option will display a number of animations with instructions to permit the user to warm up before starting the game through copying the movement displayed in the animation. The rehabilitation game option will contain the "Move-It" game that will be explained next.

The story of our game is very simple. It is about a regular person who wants to remove some colored cubes from his living room's shelf and place them into different boxes according to their color. There will be six different levels of difficulty offered in this game to maintain the user's engagement and to suit a verity of upper limb stroke patients with different severities.

This game will focus on the user's upper extremity movements, since it is mainly designed for upper limb stroke rehabilitation. It will require the patient to perform numerous arm rehabilitation movements. These movements are shoulder flexion to 90 degrees and 120 degrees to reach the cubes on the shelves, shoulder extension, abduction and adduction to move the cubes into the boxes on the right and left sides, hand extension, and grasp and release. Moreover, these shoulder movements will include implicitly the elbow flexion and extension. These movements are described by images in Table 1 and the design of the tasks that are included in the game is based on those suggested by Grimm and Gharabaghi [25]. Those tasks include grasping and movement of the arm upward and forward in different levels, which simulates daily activities that require upper limb movements.

Prior to starting the game, a tutorial that explains to the user how to achieve the game's goal is presented. At the end of each game, encouraging words and a score are displayed on the screen to motivate the user. The user statistics option contains records of the user's movement data that are presented in a simple way with graphs that the patient could easily read and understand. Moreover, these data will be synchronized to our system's database in the web server once the user selects the synchronization option, in order to be viewed later by the therapist in a web browser. It is the only option in our system that requires Internet connection to operate. Finally, the settings option enables the user to manage the game's settings such as restricting one hand and modifying profile data.

4.4. Monitoring Website. The back end of our system is the therapist monitoring website. This website will allow the therapist to remotely monitor his/her patients through viewing the patient performance records from any device that has a web browser. The therapist will enter the patient ID 
TABLE 1: Movements description (as suggested by [25]).



to retrieve his/her records. This patient ID can be similar to the patient medical record number to make establishing and memorizing these patients' ID easier for the therapies. Then (s)he can view the retrieved data by date, week, month, and so on with graphical interpretation. Moreover, the therapist can save or print these records.

4.5. User Interface Prototype. Designing our game module interfaces has followed the design guidelines for the elderly in [26] to increase the usability and accessibility of our system for older patients. The following are some of these design guidelines: (1) provide large interface elements, (2) use icons along with labels, (3) use high contrast between the interface elements, (4) provide consistent interfaces, (5) use large font size, (6) use readable font family, (7) provide good and minimum navigation, (8) always provide feedback for the user's actions, and (9) use clear and simple language. The proposed interface for the user of our game module is shown in Figure 7. On the other hand, Figure 8 represents the hierarchy of our game module.

\section{Conclusions and Future Work}

Virtual reality has been widely used in various fields, and recently it has played an important role in the rehabilitation process. In this paper we presented the steps we followed to elicit the requirements of a fully immersive virtual reality



FIGURE 7: Screenshot of the main user interface.

gaming system for upper limb stroke and provided a conceptual prototype of it. We reached this proposed design after an extensive state-of-the-art review of the field of virtual reality and serious games for upper limb stroke rehabilitation, as well as conducting patients' needs assessment.

Recent research has focused on virtual reality- (VR-) based rehabilitation systems, as they were suggested to be much effective and engaging compared to conventional rehabilitation therapy. Yet, most of these systems are nonimmersive $[5,6,8]$. Despite the fact that full-immersive systems may have the potential to provide a high level of user engagement that would solve the lack of patient interest issues, which our current proposed system will overcome, our project has 




FIGURE 8: Game module hierarchy.

included the users for this proposed system to assess their needs and incorporate them into the system design. This resulted in a list of elicited requirements to help developers in designing fully immersive virtual reality system for upper limb stroke rehabilitation.

Finally, this project is an ongoing research; the next step will be to implement our game using Unity game engine and implement and integrate the other system components. Also, we will evaluate our system using unit testing, integration testing, performance testing, and user acceptance testing to ensure that the system works correctly and to assess the user satisfaction. Furthermore, clinical pilot study will be conducted with patients and healthy subjects, to assess the effectiveness of our system.

\section{Conflicts of Interest}

The authors declare that there are no conflicts of interest regarding the publication of this paper.

\section{Acknowledgments}

This research project was supported by a grant from the "Research Center of the Female Scientific and Medical Colleges", Deanship of Scientific Research, King Saud University.

\section{References}

[1] K. Khujah, "Stroke Rehabilitation" [Online] Available: http://archive.aawsat.com/details.asp? section $=15 \&$ amp;article $=664001$ \&amp;issueno=12134\#.WHQSn1N97X5.

[2] WHO, "The world health report 2002-Reducing Risks, Promoting Healthy Life," WHO, http://www.who.int/whr/2002/en/.

[3] Alhazani, "100 stroke cases occur in SA daily," https://goo.gl/ tMzlk7.

[4] F. Alsinani, "6000 of stroke cases accure in Kingdom of Saudi Arabia yearly," 2017, http://www.alriyadh.com/56594.

[5] K. E. Laver, S. George, S. Thomas, J. E. Deutsch, and M. Crotty, "Virtual reality for stroke rehabilitation," Cochrane Database of Systematic Reviews, vol. 2, article CD008349, 2015.
[6] D. Tsoupikova, N. S. Stoykov, M. Corrigan et al., "Virtual Immersion for Post-Stroke Hand Rehabilitation Therapy," Annals of Biomedical Engineering, vol. 43, no. 2, pp. 467-477, 2015.

[7] J. W. Burke, M. D. J. McNeill, D. K. Charles, P. J. Morrow, J. H. Crosbie, and S. M. McDonough, "Serious games for upper limb rehabilitation following stroke," in Proceedings of the 2009 Conference in Games and Virtual Worlds for Serious Applications, VS-GAMES 2009, pp. 103-110, gbr, March 2009.

[8] L. Oujamaa, I. Relave, J. Froger, D. Mottet, and J.-Y. Pelissier, "Rehabilitation of arm function after stroke. Literature review," Annals of Physical and Rehabilitation Medicine, vol. 52, no. 3, pp. 269-293, 2009.

[9] P. Rego, P. M. Moreira, and L. P. Reis, "Serious games for rehabilitation: a survey and a classification towards a taxonomy," in Proceedings of the 5th Iberian Conference on Information Systems and Technologies (CISTI '10), pp. 1-6, June 2010.

[10] G. Saposnik, R. Teasell, M. Mamdani et al., "Effectiveness of virtual reality using Wii gaming technology in stroke rehabilitation: a pilot randomized clinical trial and proof of principle," Stroke, vol. 41, no. 7, pp. 1477-1484, 2010.

[11] D. Rand, R. Kizony, and P. T. L. Weiss, "Virtual reality rehabilitation for all: Vivid GX versus Sony PlayStation II EyeToy," in Proceedings of the 5th Intl. Conf. On Disability, Virtual Environments and Assoc. Technologies, pp. 87-94.

[12] Y.-X. Hung, P.-C. Huang, K.-T. Chen, and W.-C. Chu, "What do stroke patients look for in game-based rehabilitation: A survey study," Medicine (United States), vol. 95, no. 11, Article ID e3032, 2016.

[13] J. W. Burke, M. D. J. McNeill, D. K. Charles, P. J. Morrow, J. H. Crosbie, and S. M. McDonough, "Optimising engagement for stroke rehabilitation using serious games," Visual Computer, vol. 25, no. 12, pp. 1085-1099, 2009.

[14] P. J. Standen, K. Threapleton, L. Connell et al., "Patients' use of a home-based virtual reality system to provide rehabilitation of the upper limb following stroke," Physical Therapy, vol. 95, no. 3, pp. 350-359, 2015.

[15] K. J. Bower, J. Louie, Y. Landesrocha, P. Seedy, A. Gorelik, and J. Bernhardt, "Clinical feasibility of interactive motion-controlled games for stroke rehabilitation," Journal of NeuroEngineering and Rehabilitation, vol. 12, no. 1, article 63, 2015. 
[16] J.-H. Shin, H. Ryu, and S. H. Jang, "A task-specific interactive game-based virtual reality rehabilitation system for patients with stroke: A usability test and two clinical experiments," Journal of NeuroEngineering and Rehabilitation, vol. 11, no. 1, article no. 32, 2014.

[17] M. Ma and K. Bechkoum, "Serious games for movement therapy after stroke," in Proceedings of the IEEE International Conference on Man and Cybernetics, pp. 1872-1877, Singapore, October 2008.

[18] C. Kaminer et al., "An immersive physical therapy game for stroke survivors," in Proceedings of the 16th International ACM SIGACCESS Conference on Computers and Accessibility, (ASSETS '14), pp. 299-300, October 2014.

[19] S. L. Sen, Y. B. Xiang, E. S. L. Ming, K. K. Xiang, Y. C. Fai, and Q. I. Khan, "Enhancing effectiveness of virtual reality rehabilitation system: Durian Runtuh," in Proceedings of the 10th Asian Control Conference, ASCC 2015, mys, June 2015.

[20] King Fahad Medical City (KFMC), https://www.kfmc.med.sa/ EN/Pages/Home.aspx.

[21] Sultan Bin Abdulaziz Humanitarian City, Available: http://www .humanitariancity.org.sa/.

[22] Oculus Rift [Online] Available: https://www.oculus.com/rift/ [Accessed: 09-Feb-2017].

[23] Leap motion [Online] Available: https://www.leapmotion.com/.

[24] Unity-Game Engine, Unity. Available: https://unity3d.com.

[25] F. Grimm and A. Gharabaghi, "Closed-loop neuroprosthesis for reach-to-grasp assistance: combining adaptive multi-channel neuromuscular stimulation with a multi-joint arm exoskeleton," Frontiers in Neuroscience, vol. 10, 2016.

[26] B. Loureiro and R. Rodrigues, "Design guidelines and design recommendations of multi-touch interfaces for elders," in Proceedings of the ACHI, pp. 41-47, March 2014. 

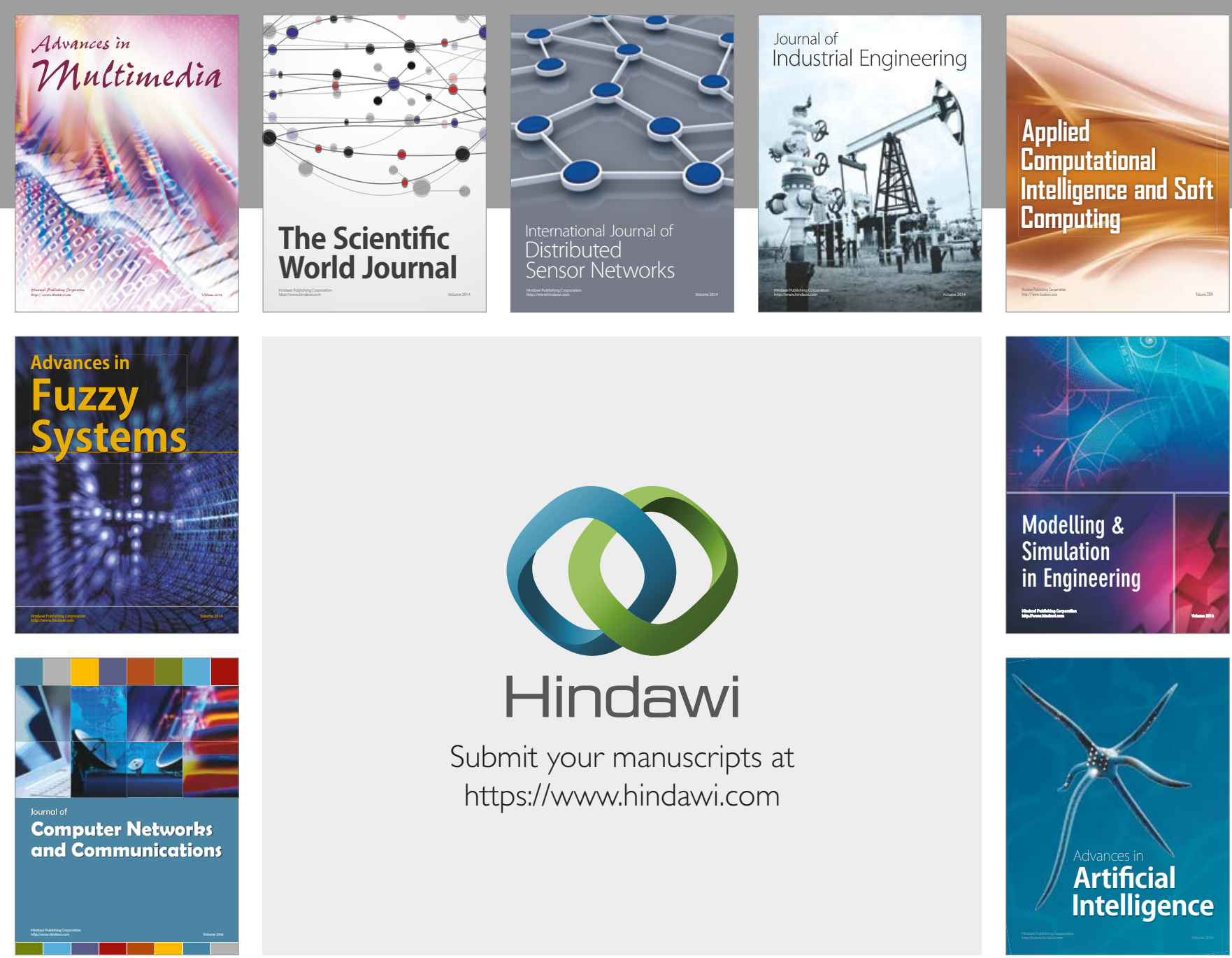

\section{Hindawi}

Submit your manuscripts at

https://www.hindawi.com
